\title{
E-Collaboration System Designed to Improve Learning Processes
}

\section{Witold Abramowicz, Tomasz Kaczmarek, and Marek Kowalkiewicz The Poznan University of Economics, Poznan, Poland}

\author{
W.Abramowicz@kie.ae.poznan.pl T.Kaczmarek@kie.ae.poznan.pl \\ M.Kowalkiewicz@kie.ae.poznan.pl
}

\begin{abstract}
Nowadays, universities are challenged by changing students' requirements, demanding labor market and fast pace environment. The evolution of communication technology allows us to deal with these problems. Advances in e collaboration are crucial to modern learning process, as it prepares students to work in groups over tasks. In this article we propose comprehensive basis for e collaboration platform which has been developed during successful implementation of e collaboration solution in Department of Management Information Systems at The Poznan University of Economics. The solution utilizes state-ofthe-art web portal technology and digital assets management system to provide consistent, common pla tform for system users to work, communicate, and share knowledge. As opposed to e learning solutions, which are designed to provide environment for distant learning, e collaboration aims at sup-porting groupwork, communication, and is rather task oriented. Therefore it is well suited for specific didactic processes in the Department. The implementation took place during two-week summer apprenticeship of 23 students in July 2002.
\end{abstract}

Keywords : e-collaboration, e-learning, document management, groupwork, portal, knowledge management, communities

\section{The Need for E-Collaboration in Non-Profit Educational Organizations}

One of the biggest challenges in today's knowledge based Internet economy is the requirement that people's knowledge and skill levels be always up-to-date. The greater the ability to create and share knowledge, the more chances on a highly competitive market an organization has. The push for constant skill improvement should be a part of an organizational culture. However, we cannot forget that organizational culture is based on experiences gained by its members even before they joined. The habit of improving ones skills should originate from at least the university level. Therefore, the new economy puts a new, demanding task on universities - to prepare its alumni for constant improvement in collaborating groups, as collaboration proves to be more efficient than individual work by introducing synergy effects, promoting creativity, tradeoffs, and - last but not least - being fun. The efforts of bringing together two or more people in order to be more creative by using electronic tools include such activities as: computer

Material published as part of these proceedings, either on-line or in print, is copyrighted by Informing Science. Permission to make digital or paper copy of part or all of these works for personal or classroom use is granted without fee provided that the copies are not made or distributed for profit or commercial advantage AND that copies 1) bear this notice in full and 2) give the full citation on the first page. It is permissible to abstract these works so long as credit is given. To copy in all other cases or to republish or to post on a server or to redistribute to lists requires specific permission from the publisher at Publisher@InformingScience.org mediated cooperation, group support systems, groupware, cooperative work, digital asset management and so on. Those efforts can be named together as e-collaboration (Kock, Davison, Wazlawick, and Ocker, 2001).

E-collaboration in non-profit educational organizations (especially at universities) leads to reducing costs in a significant number of areas of activ- 
ity, which is currently treated as a trivial statement. However, to achieve this cost reduction certain investments are required. The initial costs are significant. But after successful deployment of the solution these investments return many times. E-collaboration changes the organization by turning it into e-organization - communication between members takes place at least partially in virtual space. Moreover, e-collaboration at universities helps to improve learning process significantly. (Casanova, 2001) Several years of experiences with e-mail communication and utilization of web technology in education makes us believe it is true, thus we decided to extend current e-communication into full e-collaboration solution. It enables features which were not available for educational institutions before. Those include for instance - wider availability, instant messaging functionality, and ease of interaction. In order to communicate, one should create infrastructure. This is not always an easy task for a user. Therefore we believe that the best solution to this problem would be incorporating communication structure into the system (e.g. instant communication webpart as an element of e-collaboration portal). The mentioned features set up and justify the need for e-collaboration and therefore improve traditional educational process, which has not changed a lot for centuries. Now, with development of information systems and network technology, we are able to change traditional collaboration (e.g. teaching process) into e-collaboration, thus enriching existing interactions both between teacher and students and between students themselves. This is important step towards universities of the future. The following table (Table 1) summarizes some of the important factors that will influence future teaching.

\begin{tabular}{|l|l|}
\hline $\begin{array}{l}\text { University } \\
\text { (today) }\end{array}$ & $\begin{array}{l}\text { University - future } \\
\text { (extension of today) }\end{array}$ \\
\hline Students = young & Students = adults \\
\hline Students have no work experience & Students have significant work experience \\
\hline The teacher knows what student should learn & The student knows what she should be taught \\
\hline Teaching theories and basic skills & Teaching problem solving \\
\hline Each student attends only one university & $\begin{array}{l}\text { Distant learning - student can have many } \\
\text { classes on different universities }\end{array}$ \\
\hline
\end{tabular}

Table 1 University of today versus University of future (Cellary W. (Ed.), 2002)

These factors implicate problems that can be solved using e-collaboration. In fact, the University of Future is becoming the University of Present times. This is true to many U.S. universities, which would not be able to survive without students paying for education. However many Polish Universities - as this paper copes with Polish example - are still not ready to become "Universities of the future".

\section{Essential Elements of Successful E-Collaboration Solution}

We believe that there are at least three factors that have to be taken into consideration when planning for successful deployment of e-collaboration solution: organizational culture, scrup ulous and detailed plan, and technology.

Organizational culture can make or break the whole initiative. Introducing e-collaboration solutions in organization demands that its members accept necessity for the improvement. If they are not convinced of its advantages over current process implementation they would not accept changes. The best way to motivate and convince organization members is to let them take active part in development of planned e-collaboration project. The organization, which efficiency we plan to improve by introducing e-collaboration solution, already exists, and there is a certain community along with (clearly or unclearly) defined processes that put together this organization. The community of practice $(\mathrm{CoP})$ can and 
should be recreated. CoPs are groups of people who share information, expertise, technology, or simply take part in the same processes. They can operate at workgroup, departmental, or corporate level (Conway \& Sligar, 2002). At the educational field, we can distinguish CoPs which operate at class, departmental, school and faculty level. Less often, there are groups which function at cross- university level. Ecollaboration requires extending this community into virtual community. Evolution of organization demands that we first ,virtualize" its existing structures and only after that can we introduce new ones. This leads to modification of organization based on experiences gained through e-collaboration. E-collaboration develops new connections between community members while still supporting and enhancing those already existing. It eases building new connections as it is easy to find people that have similar interests or goals. Such community members gather around certain newsgroups, chats and mailing lists, which are available only to community members, in order to exchange ideas. This communication means tightening relations in communities that already exist in educational organizations. Becoming a member of these groups is relatively easy for people that are too much intimidated to join more formal groups (Walker, Ilardi, McMahon, and Fennell). Therefore, it eases integration of student groups. This is true nowadays, when studying is tightly bound with joining students' community and becoming member of several groups. However in the future peoples attitude towards learning can change. New techniques of communication and demanding labor market may shift students' interests from gaining formal education to achieving certain skills. They may become more information than socially oriented. Nevertheless it is hard to deny that instant messaging eases communication. Moreover, it changes people's attitude towards communication. People tend to be more creative and communicate more often as opposed to traditional face-to-face communication where shy people are often inhibited by more straightforward group members (Garton and Wellman, 1993). There can of course be the opposite effect - some may easily communicate verbally while having trouble expressing their thoughts in writing. Though, they also may benefit from instant messaging even with its current limited capabilities.

E-collaboration requires also some of the processes to be redesigned. This includes formal definitions of processes and its participants, simplification, and sometimes introduction of new processes demanded by information system. The example are processes related to maintaining user information in the system, user identification, granting permissions, administration of user personal and public resources stored in the system.

Thoroughly planned solution - a broader view on a project. After being convinced of the need of e-collaboration one has to define the outcome of the whole transformation. It means that before starting to implement it is necessary to define borders of the solution and think over what should be contained between these borders in detail. This is crucial for defining tasks to be carried out during implementation. However the success depends both on social and technological aspects. The former are related to organizing a group of people into e-community and managing the group. One has to clearly state:

1. The purpose for establishing a community.

2. Community members.

3. Membership process.

4. Community rules.

5. Member generated content.

Technological aspects that have to be taken into account include:

1. The area to be covered by e-collaboration.

2. User information and rights.

3. Process design. 
4. Data organization.

5. Data flow and workflow:

a. Data organization.

b. Processes design and implementation.

6. Means to access data and work in "e"-space.

Technology. The technology itself cannot guarantee success - a common mistake is to focus on technical aspects while neglecting preparation phase or social factors. The essential technological elements were described in previous paragraphs, here it seems enough to say that recent advances in network technology and still growing number of means of communication (WWW, Newsgroups, Email, Instant Messaging, VoIP, and Videoconferencing) form just right environment for e-collaboration.

\section{State-of-the-Art}

According to the researches (Kock, Davison, Wazlawick, and Ocker, 2001), the e-collaboration phenomenon dates back to 1970 s, when the first electronic information exchange system (EIES) was proposed. One of its creators suggested few years later a new approach to videoconferencing by using the web for this purpose. Late 1980s and mid 1990s were the best years for e-collaboration technology, as by the mid 1990s a significant number of e-collaboration applications hit the market. Many of them (such as Lotus Notes or Peoplesoft) are still very popular.

In the field of traditional education there are, however, not many dedicated e-collaboration products. Major software vendors have tried to introduce their products, without significant success. Therefore we believe that the topic of creating e-collaboration solutions for didactic ground is still a place for full-time Einstein.

\section{Technologies to Support E-Collaboration in Educational Organizations}

In this paper the authors propose implementing an e-collaboration platform which aims to improve didactic process at universities. This is based on real experiences gained during planning and implementation phases, followed by real-life tests during university courses at the Poznan University of Economics in Poland.

The solution we propose does not go directly towards Virtual University (VU) paradigm. By introducing the platform we want to enhance traditional didactic process, which proves to be successful, as it has proved to be for hundreds of years until now. Of course, we should not forget that emerging VU solutions are an answer to the problem of people being able to study only remotely. However, our proposal does not deal directly with distance learning issue. Nevertheless, the platform can be incorporated in a larger distance learning project that will provide additional required features.

As it was declared, technology should never be considered as a crucial element for building e-collaboration solution. Nevertheless, by planning for appropriate technological mixture, one can more easily put the two remaining aspects (organizational culture and thoroughly planned solution) into practice. From the technological point of view, our proposed set of ingredients is as follows: network server, directory service, messaging server, instant messaging server, digital asset management system, and web portal.

Network server. The base for e-collaboration solution should be established on a network server, which can take advantage of organization's internal knowledge resources (presumably file servers, data warehouses, databases, and document repositories). Therefore, such a server should support at least TCP/IP 
protocol and other protocols used internally by an organization (such as IPX/SPX or NetBIOS). However, we opt for storing corporate knowledge in one place (we purposefully omit security considerations here), one format (or with standard metadata descriptions), accessible in the same way (by using the same network protocol). At the same time, the network server should be able to get through to external knowledge and information resources (structured and semi-structured) in order to respond to user requests. This should be done by using web services features based on well accessible UDDI descriptions. Leading public retrieval systems provide web service interfaces to their engines (Dornfest, 2002).

Directory service. One should try to reuse most of existing data, and make new data, which is entered into the system, available to others. Hence, the e-collaboration solution should store all, or most of, user related data in well known structures, such as standard directory services (Novell Directory Services, Active Directory or Lightweight Directory Access Protocol, just to name a few). Apart from storing users' contact and configuration information, this should also include storing information about user groups, user rights, available services etc.

Messaging server. One of most important cooperation aids, even in relatively low dispersed environment is communication software. This includes email server as well as more advanced newsgroup servers. Such complex systems are available for all major operating systems.

Instant messaging server. It is often required that an individual contacts another one in order to discuss some topic, exchange ideas or train each other. Instant messaging server can be helpful in such situation. This enables to create a one-on-one chat room, often sound and video enhanced. The typical instant messaging server alerts the user whenever somebody on her private list is on-line. Unfortunately, there is no standard protocol in messaging systems, and there is a large number of competing instant messaging systems.

Digital asset management system (DAM) is a central repository for organization's digital files (documents, images, streaming videos etc.). This should also be a central point of storage for e-collaboration solution. There are many competitive technologies to be used, such as relational databases, data warehouses, or specialized products offered by software vendors. The key cha racteristics when choosing the proper DAM are: performance, flexibility, and open standards compatibility.

Web portal. In order to provide a standard and well accessible user environment, one can decide to use web portal technology. A standard web portal offers such services as e-mail, search engine, forums, news etc. For that reason authors believe that portal should also be used for e-collaboration purposes.

\section{Department of MIS Case Study}

Organizational culture. The Department of Management Information Systems at The Poznan University of Economics, Poland, offers education in a field of business information processing. The department concentrates on information retrieval and filtering for Management Information Systems, data warehouses, workflow, e-marketplace and mobile systems. Its members also conduct research on decision support systems for top-level management of a company and for investment activities. Moreover, its interests cover other fields of business information like personalization of Internet services, knowledge management systems, customer relationship management systems and more. One of more popular didactic methods used in the department is writing essays at the end of each semester. Since this practice has been in use for a few years, the essay library has grown large - up to several hundred of CDs (each disc contains an essay, PowerPoint presentation and resources - mainly documents downloaded from the Web during the essay preparation). Such library in its form has become unmanageable, and could not be efficiently used by students and departmental researchers. On the other hand, one should not underestimate the value of the library - thousands of documents chosen by potential experts in the field along with essays, most of them containing valuable knowledge. 
Scrupulous and detailed plan. The group of departmental researchers who focus on knowledge management decided to implement an e-collaboration system for the department basing on a digital asset management system. This is a part of a larger scale project, named D-Leap aiming to implement a full knowledge management life cycle using topic maps and skill maps (Abramowicz, Kowalkiewicz \& Zawadzki, 2002) in fully personalized courseware distribution. The deployment plan included moving existing communities into the e-collaboration platform, full support for didactic process - from the first year of studies until the last contact with Department (including PhD theses), and complete project of knowledge base structure, along with user access rights. Throughout the tests it seems, that the plan accurately predicted the needs.

The platform has been created using three server machines: directory services server, portal server and DNS server, all of them in the same Internet domain: kie.ae.poznan.pl. DC and portal are Windows 2000 Server based systems, DNS runs on Sun's SunOS. The portal server utilizes Microsoft's SharePoint Portal Server and document repository in Web Storage System. DC is a primary Windows Domain Controller (Active Directory server) and an Exchange server, Sun's machine is a primary name server for the whole Internet domain.

These servers cooperate in order to produce common environment for system users. The front end portal is delivered by Microsoft's SharePoint Portal Server which is also digital assets management system. The document repository has hierarchical structure, defined according to topics of interests and categories. Document management capabilities of SPS have been extended to support educational process needs. Web Part technology used to create portal interface allows integrating such modules as search engine, instant messaging and email access, advanced portal navigation through categories, subscriptions management (users can select portal areas in order to be emailed of their content changes). The backend of the system is provided by Active Directory and Exchange 2000 server. Directory service is accessed by scripts run on web server to store user information and manage user rights (in combination with SPS security system). It is also a database of user information for Exchange server which stores user mailboxes, enables instant messaging and group emailing.

The system has been implemented with help of full-time university students during summer apprenticeship in July at the Poznan University of Economics. Twenty one students and two research assistants built a team, made of six groups: developers 1, developers 2, administrators, knowledge engineers, UI designers, and managers. The total workload was 1590 hours during two weeks of intensive work (preceded by two weeks of system planning), which gives approximately 7.6 hours of intensive work per day per participant. The developers focused on programming new features (mainly webparts) for SharePoint Portal Server. New features include:

- full Active Directory integration

- Microsoft Exchange and Outlook Web Access available directly on the portal

- class registration

- essay authoring (with teacher's acceptation) workflow implementation

- essay deadline control

- student group management

- teacher and lecturer group management

- document repository management - structure control

Features delivered by our e-collaboration system that support educational process incorporate messaging, wide availability of content and groupwork - as mentioned earlier. These are to be tested during the winter semester 2002 by several groups of students. The environment that we prepared is designed to 
help them gather materials, complete tasks that were assigned to small groups and communicate within them. It helps teachers to coordinate students' efforts and easily communicate with groups.

The system supports e-collaboration providing storage and contact place for distant users. Through publication capabilities it enables cooperation in creating added value. For example a temporary website was established during system implementation to support and coordinate efforts of different project groups. The system is also used for department project coordination and document creation. This paper has been written using the departmental platform. The authors didn't have to meet face-to-face, as every new version with comments appeared on the system. The check-in - check-out feature helped to maintain one version of document (at no time had two authors worked on a document without knowing of it). The subscription notification informed authors if the new version of a document turned up on the platform.

\section{Conclusions}

In today's world, successful implementation of e-collaboration systems in universities is a must. It not only reduces costs, but - more importantly - prepares alumni to compete successfully on labor market. It also prepares students for lifelong learning, which seems to be the key to success in forthcoming years. We believe that while planning the e-collaboration deployment, one should take into consideration three parts of a project: organizational culture, scrupulous and detailed plan, and technology. Because there are not many e-collaboration products on the market, that provide complete answer to universities needs, there is a great potential in creating individual implementations. Such implementation, basing on the assumptions described in chapters 2 and 4 has been created at the Department of Management Information Systems, The Poznan University of Economics (chapter 2). The system is now undergoing tests, but results are promising.

\section{Acknowledgements}

The authors would like to thank the students who volunteered in the project preparation during summer apprenticeship:

- Administrators: Wojciech Zalech (group manager), Tomasz Baumgart, Tomasz Jakubowski, Wlodzimierz Piatek, Pawel Zebrowski

- Developers 1: Piotr Kolodziej (group manager), Agata Godlewska, Wojciech Rutkowski

- Developers 2: Lukasz Kobusiewicz (group manager), Jacek Kopcinski, Marek Mizier, Piotr Slowikowski

- Knowledge engineers: Agata Filipowska (group manager), Jakub Abram, Julia Gwizdala, Monika Kaczmarek, Andrzej Kyc, Pawel Pisarczyk, Dominik Zyskowski

- UI designers: Robert Dziadosz, Jacek Waszak

We would also like to thank Microsoft Poland for their support and providing software that could be used for implementing the system, and therefore providing a basis for further e-collaboration and e-learning researches.

\section{References}

Abramowicz W., Kowalkiewicz M., Zawadzki P. (2002). Tell me what you know or I'll tell you what you know. Skill map ontology for information technology courseware. Issues \& Trends of Information Technology Management in Contemporary Organizations. pp. 7-10.

Casanova A. (2001). CMC Technologies: Learning in an E-Collaborative Classroom. WVNET User Conference 2001 Proceedings. Retrieved September 21, 2002, from http://tux.wvnet.edu// sarah/conf2001 docs/CMCTech.htm 
E-collaboration system designed to improve learning processes

Cellary W. (Ed.), Abramowicz W. et al. (2002). Polska w drodze do Globalnego Spoleczenstwa Informacyjnego, Raport o rozwoju spolecznym, Program Narodów Zjednoczonych ds. Rozwoju, Warszawa 2002. Retrieved September 25, 2002 from http://www.kti.ae.poznan.pl/nhdr2001/

Conway S., Sligar C. (2002). Unlocking knowledge assets. Redmond: Microsoft Press.

Dornfest R. (2002, April 11). Google Web API. The O'Reilly Network. Retrieved September 16, 2002, from http://www.oreillynet.com/lpt/wlg/1283

Garton, L. E., and Wellman, B. Social Impacts of Electronic Mail in Organizations: A Review of the Research Literature, Toronto, Canada: University of Toronto, Centre for Urban and Community Studies and the Ontario Telepresence Project, 1993 Retrieved September 23, 2002, from: http://www.dgp.toronto.edu/tp/papers//9313.html

Kock, N., Davison, R., Wazlawick, R. and Ocker, R. (2001), E-Collaboration: A Look at Past Research and Future Challenges. Journal of Systems and Information Technology, V.5, No.1 . pp. 1-9. Retrieved September 18, 2002, from http://www.mis.temple.edu/kock/Publications//2001JSIT/eCollabIntro02.htm

Utz, S. Social Information Processing in MUDs: The Development of Friendships in Virtual Worlds. (2000). Journal of Online Behavior (1:1). Retrieved from: http://www.behavior.net/JOB/v1n1/utz.html

Walker, H. A., Ilardi, B. C., McMahon, A. M., and Fennell, M. L. (1996). Gender, Interaction, and Leadership, Social Psychology Quarterly (59:3), pp. 255-272. 